\title{
Analysis of the strengthening and toughening of a biomaterial interface
}

\author{
SONG Fan (宋 凡) \& BAI Yilong (白以龙)
}

LNM, Institute of Mechanics, Chinese Academy of Sciences, Beijing 100080, China

Correspondence should be addressed to Song Fan (email: songf@ Inm.imech.ac.cn)

Received January 16, 2001

Abstract Based on the transmission electron micrographs of nacre, the existence of mineral bridges in the organic matrix interface is confirmed. It is proposed that the microarchitecture of nacre should be considered as a "brick-bridge-mortar" (BBM) arrangement rather than traditional "brick and mortar" (BM) one. Experiments and analyses indicate that the mineral bridges effectively affect the strength and toughness of the interfaces in nacre. Comparison with a laminated composite with BM structure, SiC/BN, shows that the pattern of the crack extension and the toughening mechanism of the two materials are different. This reveals that the mineral bridges play a key role in the toughening mechanisms of nacre, which gives a conceptual guidance in material synthesis.

Keywords: organic matrix interface, mineral bridge, nacre, microstructure, fracture toughness.

Nacre is a natural platelet-reinforced composite. Its traditional model is considered as a "brick and mortar" (BM) arrangement, where brick refers to flat polygonal crystals of aragonite, and mortar, the organic matrix interface (sheet) in nacre, is a biological organic adhesive composed of polysaccharide and protein fibers ${ }^{[1-8]}$. In recent years, a great deal of attention has been paid to the microarchitecture of nacre due to its excellent mechanical properties ${ }^{[9-11]}$. Previous work indicated that the strength and tough mechanisms of nacre are determined by its microarchitecture $^{[2-7]}$, which, however, has not yet been identified clearly ${ }^{[2-4]}$. Recently, some researchers ${ }^{[5,6,12]}$ suggested that there might be a number of mineral bridges in the interface, as they found many pores in the mortar sheets of nacre. However, they could not offer the conclusive evidence of the mineral bridges ${ }^{[8,12,13,15]}$.

On the other hand, to investigate the excellent mechanical performance of nacre (for example, an abalone nacre has a work of fracture about 3000 times greater than that of a single crystal of the pure mineral ${ }^{[4,5]}$ ), several groups have studied the relationship between the mechanical properties and microarchitecture of nacre ${ }^{[1-3]}$. However, the possible strengthening and toughening mechanisms based on the microarchitecture of nacre are not well understood ${ }^{[1-3]}$. For example, Smith et al.$^{[4]}$ believed that the key to nacre's fracture resistance resides in the polymer adhesiveness of the organic interface. Currey ${ }^{[1]}$, Jackson et al. ${ }^{[2,14]}$ and Vincent ${ }^{[7]}$ studied the strength and toughness of nacre based on the BM structure and indicated that the governing factor is the microstructures of the material. In addition, synthetic biomimetic materials with BM structure do not have a toughness comparable to nacre ${ }^{[4,13]}$.

In the present work, a direct observation of mineral bridges in the organic matrix interface of nacre is carried out. By virtue of mechanical experiments and analyses, we demonstrate that the mineral bridges can effectively enhance the stiffness, strength and toughness of the interface. In 
addition, we compare nacre with synthetic biomimetic materials, and indicate that the pattern of the crack extension in nacre is significantly different from that of the synthetic materials with BM arrangement. Our investigations show that the existence of mineral bridges causes the difference between the fracture patterns of the two materials. However, the predicted length of crack extension in the synthetic materials, according to the analyses on nacre, is in agreement with the experimental. This reveals the contribution of mineral bridge structure to mechanical properties of the materials, thus giving a conceptual guidance to the biomimetic design of synthetic materials .

\section{Microarchitecture and mineral bridges}

The testing samples of nacre are from Paua shell, an abalone shell from New Zealand. This nacre is a ceramic composite containing $95 \%$ of interlocking aragonite tablets staggered in successive laminae and separated by a $5 \%$ organic matrix. At ambient temperature, the water content at saturation is $0.2 \%$ of the total mass of fresh nacre.

Direct observation of the nacre with the TEM shows that the cross sectional surface reveals an aptitude for a BM structure (fig. 1). The thickness and width of an aragonite platelet in the cross sectional surface of nacre are $0.37-0.40 \mu \mathrm{m}$ and $1.8-4.6 \mu \mathrm{m}$, respectively. The thickness of an organic matrix interface is $25-32 \mathrm{~nm}$. However, there do exist many mineral bridges in the organic matrix interfaces. The the mineral bridges look like circular columns and randomly distriute in the organic sheets. The diameter of a mineral bridge is $38-50 \mathrm{~nm}$, and the height of a mineral bridge is equal to the thickness of the organic interface, $25-32 \mathrm{~nm}$. The density of mineral bridges on an aragonite platelet layer is $91-116 \mu \mathrm{m}^{-2}$. Therefore, the microarchitecture of nacre should be referred to as a BBM structure instead of BM one. It has also been report$\mathrm{ed}^{[13,14]}$ that the crystallographic orientations of 3-10 successive platelets on a cross section of nacre can remain the same. So all these observations may suggest a slightly tangled BBM structure.
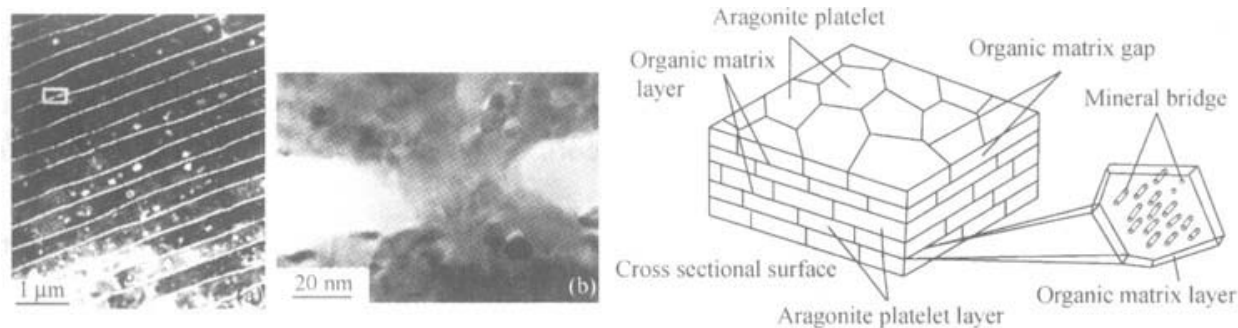

(c)

Fig. 1

\section{Effects of mineral bridges on strength}

In the analyses of the mechanical properties of nacre, we assume that an interlamellar organic sheet is fetched out of two successive aragonite platelets (fig. 1(c)). Obviously, the mechanical behaviors of the organic matrix sheet are anisotropic due to the microarchitectures of nacre. A surface area of the organic interface on a platelet is approximately $16 \mu^{2}$, while the total cross sectional area of the mineral bridges in the organic sheet is about $2.7 \mu \mathrm{m}^{2}$. Since the area of the latter is about one sixth that of the former, it is reasonable to treat the organic sheet as a fiber reinforced composite in which the matrix is organism and the fibers are mineral bridges (fig. 1 
(c)). We approximately take the Young's moduli of the mineral bridges and organic matrix as $E_{\mathrm{b}}=100 \mathrm{GPa}$ and $E_{\mathrm{o}}=4 \mathrm{GPa}$, respectively ${ }^{[5]}$, and the volume fractions of the fiber and matrix of the composite as $V_{\mathrm{b}}=1 / 6$ and $V_{\mathrm{o}}=5 / 6$, respectively. According to composite theory, we have

$$
\begin{aligned}
& \frac{E_{\mathrm{c}}}{E_{\mathrm{o}}}=V_{\mathrm{b}}=\frac{E_{\mathrm{b}}}{E_{\mathrm{o}}}+V_{\mathrm{o}}=5, \\
& \frac{E_{\mathrm{c}}^{*}}{E_{\mathrm{o}}}=\frac{1}{V_{\mathrm{o}}+V_{\mathrm{b}} \frac{E_{\mathrm{o}}}{E_{\mathrm{b}}}}=1.2,
\end{aligned}
$$

where $E_{\mathrm{c}}$ and $E_{\mathrm{c}}^{*}$ are the Young's modulus of the composite in the direction of the mineral bridges and in the organic matrix layer, respectively. This indicates that the mineral bridges increase the stiffness of organic sheet in the direction of the mineral bridges by five times. Obviously, if $V_{\mathrm{o}}=1$ and $V_{\mathrm{b}}=0$, then $E_{\mathrm{c}}=E_{\mathrm{c}}^{*}=E_{\mathrm{o}}$; that is, the organic matrix layers are isotropic, and such a model becomes traditional BM model.

Both the tensile and the three-point bending tests of dry nacre show that the strain of organic matrix sheets in direction of mineral bridges is approximately linearly elastic and very small. Since the fraction of the mineral bridges in the interface is about one sixth, the strength of the composite is controlled by the organic matrix. From the strength theory of composite, we have

$$
\sigma_{\mathrm{c}}=\sigma_{\mathrm{o}}\left(V_{\mathrm{b}} \frac{E_{\mathrm{b}}}{E_{\mathrm{o}}}+V_{\mathrm{o}}\right)=5 \sigma_{\mathrm{o}},
$$

where $\sigma_{\mathrm{c}}$ and $\sigma_{\mathrm{o}}$ are the strength of the composite and organic matrix. It is shown that mineral bridges enhance the strength of organic matrix layers by five times.

\section{Effects of mineral bridges on crack resistance and fracture toughness}

\subsection{Crack resistance}

We performed the tension and three-point bend tests on the nacreous samples (fig. 2(a) and $(b))$, and found in the tests that (i) the strain of organic matrix in the direction of mineral bridges is approximately linearly elastic and very small before crack extension; and (ii) all cracks only extend in the interlamellar organic layers of nacre.

Considering the above fiber reinforced composite of nacre, the average crack resistance on one organic matrix sheet can be written as
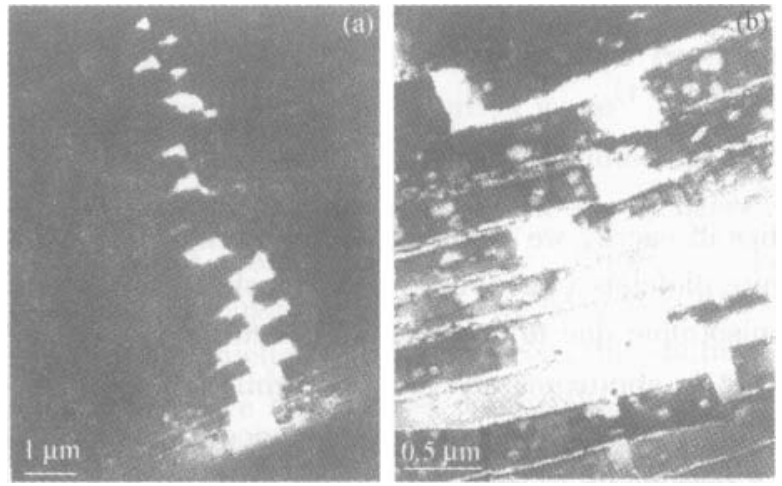

Fig. 2

$$
R_{\mathrm{c}}=2 \gamma_{\mathrm{o}} V_{\mathrm{o}}+2 \gamma_{\mathrm{b}} V_{\mathrm{b}}+2 \gamma_{\mathrm{o}} \pi D t \rho,
$$

where $\gamma_{o}$ and $\gamma_{b}$ are the surface energies of the interface and mineral bridges, respectively. $D=46 \mathrm{~nm}$ is the diameter of mineral bridges, $t=29 \mathrm{~nm}$ is the height of mineral bridge and $\rho=100 \mu \mathrm{m}^{-2}$ is the density of mineral bridges on the aragonite platelet. In eq. (4), $\pi D t \rho \gamma_{0}$ stands for the surface energy induced by the interactions between the mineral bridges and organic matrix on an aragonite platelet. By using the average 
thickness of an organic matrix layer $t=29 \mathrm{~nm}=29 \mathrm{D} / 46$, and neglecting the contribution of the fracture surface energy of the bridges $2 \gamma_{b} V_{b}$, we approximately give the average crack resistance as

$$
R_{\mathrm{c}} \approx 2 \gamma_{\mathrm{o}}\left(1+1.5 V_{\mathrm{b}}\right)=R_{\mathrm{o}}\left(1+1.5 V_{\mathrm{b}}\right),
$$

where $R_{0}=2 \gamma_{0}$ stands for the crack resistance of the organic matrix layer without the mineral bridges. With $V_{\mathrm{b}}=1 / 6$, we have $R_{\mathrm{c}}=1.25 R_{\mathrm{o}}$; that is, the mineral bridges increase the average crack resistance by 1.25 times.

\subsection{Fracture toughness}

In terms of the fracture toughness in the interface of nacre, when the effects of mineral bridges are not considered, we write the fracture toughness of the organic matrix sheet on one platelet as

$$
K_{1}^{\mathrm{o}} \mathrm{c}=\sqrt{E_{\mathrm{o}} R_{\mathrm{o}}}
$$

when the existence of mineral bridges is considered, we obtain the fracture toughness in the interface of the composite

$$
K_{1 C}^{\mathrm{c}}=\sqrt{E_{\mathrm{c}} R_{\mathrm{c}}} .
$$

Using eqs. (1) and (5), we obtain

$$
K_{1 C}^{\mathrm{c}}=K_{1 C}^{\mathrm{o}} \sqrt{5\left(1+1.5 V_{\mathrm{b}}\right)} \approx 2.5 K_{1 C}^{\mathrm{o}} \text {. }
$$

Obviously, the mineral bridges enhance the toughness of the interfaces by 2.5 times.

\subsection{Crack stopping and length}

It might be interesting to tackle a problem discussed widely: why does the crack stop after extending a distance in an interlamellar organic matrix sheet ${ }^{[4,5]}$ ? Firstly, the experimental data statistics show that the mean length of the crack extension distribution is $1.2 \mu \mathrm{m}$ on cross sectional surface of nacre. It is about three times the thickness of an aragonite platelet and much less than the mean width of the aragonite platelets on the cross sectional surface, $3.4 \mu \mathrm{m}$, implying that mineral bridges prevent the crack extension in the organic matrix interface. Secondly, according to the theory of fracture mechanics, we write the fracture toughness of materials as

$$
K_{1 c}=\sigma \sqrt{\pi a},
$$

where $\sigma$ is the strength of material and $a$ is half the length of crack. Applying eqs. (2)-(4) to (5) yields

$$
\frac{a_{\mathrm{c}}}{a_{\mathrm{o}}}=2.5^{2}\left(\frac{\sigma_{\mathrm{o}}}{\sigma_{\mathrm{c}}}\right)^{2}=\frac{1}{4},
$$

where $a_{c}$ and $a_{o}$ are half the length of crack in the organic matrix sheets with and without mineral bridges, respectively. Eq. (6) indicates that the length of the crack extension in an organic sheet without the mineral bridges is four times greater than that of a real crack, i.e. 12 times the thickness of the platelet on the surface, showing that the mineral bridges effectively hinder the crack extension in the organic interfaces .

\section{$4 \mathrm{SiC} / \mathrm{BN}$ testing}

To validate the above analyses, we examined the laminated composites with BM structure whose matrix layer is $\mathrm{SiC}$ and weak interface is $\mathrm{BN}$ on a Hitachi S-570 SEM. The SiC and BN were 200 and $25 \mu \mathrm{m}$ thick respectively. The fracture surface morphologies of the synthetic materi- 
als are shown in fig. 3(a) and (b). It can be observed that the crack development is also tortuous, but crack extension in the weak interfaces is sufficient and much longer than that of nacre . In addition, there exist the parts of sample breaking away in the course of fracture (fig. 3(b)) . The mean distance of the crack extension in the weak interfaces was approximately equal to 13 times the thickness of $\mathrm{SiC}$ layer. This is very approximate to the predicted result on nacre that the length of crack extension in the organic sheet without the mineral bridges is 12 times the thickness of the aragonite platelet. Our analyses show that the difference between the predicted and the experimental results stem from the following facts: (i) the structural scale of the synthetic material is much larger than that of nacre; and (ii) the $\mathrm{SiC}$ layer is not divided into tablets.

In fact, platelet pull-out and crack de-

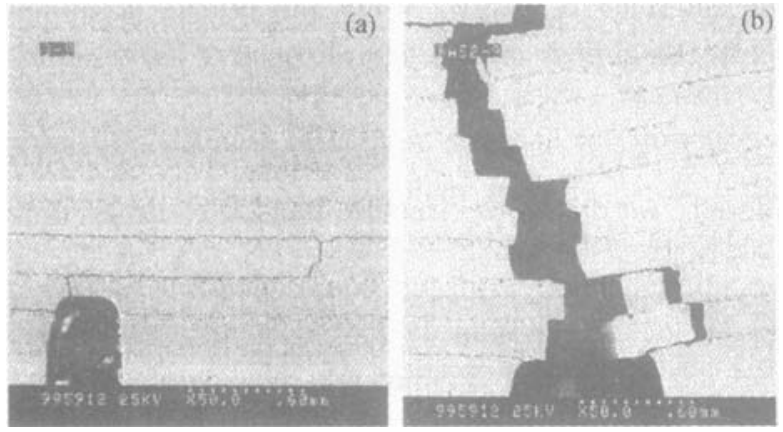

Fig. 3 flection are two common toughening mechanisms found in dry nacre and synthetic platelet-reinforced ceramics ${ }^{[6,12]}$, and it has been recognized that a weak matrix interface is very important for the two mechanisms. Therefore, many attempts have been made to obtain an appropriate interface so that not only the interface can gain sufficient strength but also a crack can be easily deflected at the interface. From the viewpoint of interfacial design, nacre is an ideal composite, which is composed of inorganic ceramic phase (aragonite) and the organic matrix organized in an ordered way. The organic matrix between aragonite layers is weak relative to its ceramic component. Since mineral bridges exist in the organic sheets, the weak interface in nacre is reinforced, suiting a crack to propagate along the organic matrix rather than to penetrate the hard phase. As far as the two sorts of material microstructure are concerned, it is mineral bridge that induces the different forms of crack extension in the weak interface. On the other hand, from mechanical point of view, it may be this distinction that makes the strength and toughness of nacre much higher than that of synthetic materials with BM structure.

\section{Conclusions}

Via direct observation by virtue of TEM, we confirm the existence of mineral bridges in the organic matrix interface of nacre. Based on the mechanical experiments and analysis, we have studied the stiffness, strength and toughness of the interface and demonstrated that the effects of the mineral bridges on the organic matrix interface of nacre are important. The excellent toughness of this biomaterial is the result of two toughening mechanisms acting in concert: crack deflection and platelet pull-out. These toughening mechanisms are closely associated with the mineral bridges in organic matrix interface, and are direct results of the unique microstructure of nacre. That is why synthetic biomimetic materials with BM structure do not have a toughness comparable to nacre.

Acknowledgements This work was supported by the National Natural Science Foundations of China (Grant Nos. 19891180 and 10072067) and the Natural Science Foundation of Chinese Academy of Sciences (KJ951-1-201). 


\section{References}

1. Currey, J. D., Mechanical properties of mother of pearl in tension, Proc. R. Soc. Lond. B, 1977, $196: 443-463$.

2. Jackson, A. P., Vincent, J. F. V., Turner, R. M., The mechanical design of nacre, Proc. R. Soc. Lond. B., 1988, $234: 415-440$.

3. Wang, R. Z., Wen, H. B., Cui, F. Z . et al., Observations of damage morphologies in nacre during deformation and fracture, J. Mater. Sei., 1995, 30: 2299-2304.

4. Smith, B. L., Schaffer, T. E., Viani, M. et al., Molecular mechanistic origin of the toughness of natural adhesives, fibres and composites, Nature, 1999, 399: $761-763$.

5. Addadi, L., Weiner, S., A pavement of pearl, Nature, 1997, 389: 912-915.

6. Schaffer, T. E., Ionescu-Zantti, C. , Fritz, M. et al., Does abalone nacre form by heteroepiaxial nucleation or by growth through mineral bridges? Chem. Mater., 1997, 9: 1731-1740.

7. Vincent, J. F. V., Structural Biomaterials, New York; The Macmillan Press Ltd, 1982.

8. Song, F., Bai, Y. L., Effects of mineral bridges on the mechanical properties of nacre, Acta Mechanica Solida Sinica (in Chinese), 2000, 21 (Special Issue): $171-176$.

9. Szuromi, P., Microstructural engineering of materials, Science, 1997, 277: 1183-1183.

10. Heuer, A. H., Fink, D. J., Laraia, J. L. et al., Innovative materials processing strategies ; a biomimetic approach, Science, 1992, 255: 1098-1105.

11. Stupp, S. I., Braun, P. V., Molecular manipulation of microstructure: biomaterials, ceramics, and semiconductors, Science, 1997, 277: 1242-1248.

12. Watabe, N., Crystal growth of calcium carbonate in the invertebrates, Prog. Crystal Growth Charact., $1981,4: 99-147$.

13. Clegg, W. J., Kendall, K., Alford, N. M. et al., A simple way to make tough ceramics, Nature, 1990, 347: 455-457.

14. Jackson, A. P., Vincent, J. F. V., Briggs, D. et al., Application of surface analytical techniques to the study fracture surface of mother-of pearl, J. Mater. Science Letters, 1986, 5: 975-980.

15. Feng, Q. L., Su, X. W., Ciu, F. Z. et al. , Crystallographic orientation domains of flat tablets in nacre, Biomimetics, $1995,3: 159-167$. 